\title{
Does Chemo-Radiation Therapy Influence Outcomes in Unresectable Locally Advanced State IV Rectal Cancer?
}

\author{
Joaquin J. Estrada, Vivek Chaudhry, Jose R. Cintron, Leela M. Prasad, Herand Abcarian \\ Division of Colon and Rectal Surgery, John H. Stroger Jr. Hospital of Cook County, Chicago, USA \\ Email: abcarian@uic.edu
}

Received August 15, 2012; revised September 20, 2012; accepted September 30, 2012

\begin{abstract}
Introduction: The median survival for patients with stage IV rectal cancer is approximately 20 months. Therapy should focus not only on improving survival but also on quality of life. The aim of our study was to determine if chemoradiation (C-RT) would improve palliation for metastatic unresectable locally advanced disease compared to patients receiving palliative chemotherapy alone (C) for stage IV cancer. Methods: Retrospective review of a prospectively maintained database at a single institution was carried out under IRB approval. From January 2004 to December 2008, 43 patients presenting with unresectable stage IV rectal cancer were identified with a median follow-up of 12 months. Patients with evidence of locally advanced disease or bulky disease received infusional 5-FU \pm bevacizumab and 3D conformed mega voltage photon therapy (5400 cGy). Patients without evidence of bulky disease received either FOLFOX or FOLFIRI \pm bevacizumab. Data on demographics, investigations, treatment, complications, metastasis, number of blood transfusions, days of hospitalization, and surgical intervention were analyzed using SPSS statistical software. p < 0.05 was considered statistically significant. Results: There were 25 and 18 patients in the C and C-RT groups respectively. There was no difference in mean age, sex or overall survival. Three patient (12\%) in the C group developed hydronephrosis compared to 8 patients $(44 \%)$ in the C-RT group (p < 0.05). Six patients (24\%) developed bowel obstructions requiring an ostomy in the $\mathrm{C}$ group compared to 9 patient (50\%) in the C-RT group (p = 0.07). In the C arm, 80\% of patients required multiple hospitalizations for symptoms consistent with progression of disease compared to $61 \%$ of patients in the C-RT arm $(\mathrm{p}<0.01)$. Conclusion: Chemoradiation in patients with locally advanced unresectable stage IV cancer has not been extensively investigated. At our institution, patients treated with C-RT for bulky stage IV rectal cancer required fewer hospitalizations when compared to those treated with chemotherapy alone.
\end{abstract}

Keywords: Chemo-Radiation; Cancer

\section{Introduction}

Despite well delineated screening protocols, colon and rectal cancer remains the 3rd most commonly diagnosed malignancy in the United States [1]. Approximately 140,000 patients were diagnosed with a colorectal cancer in 2010 [1,2] and over 39,000 of them had rectal cancer [2-4]. Current treatment strategies for rectal cancer are based on clinical staging. The majority of patients present with resectable disease [5] and treatment algorithms may include surgery alone for proximal rectal cancer vs. multimodality approach (chemotherapy, radiation therapy and surgical resection) for mid-distal rectal cancer.

R0 resections are necessary for favorable long-term outcomes [6-8]. In patients diagnosed with locally advanced or unresectable metastatic disease R0 resection is frequently not possible. As a result, survival rates are dismal. Historically, the median survival for stage VI rectal cancer has been 7 - 12 months [9]. However, with advancement in chemotherapy regiments and the addition of mono-clonal antibodies the survival has been extended to approximately 20 months [4].

Unfortunately, with this advanced state of disease patients frequently require multiple hospitalizations for the management of gastro-intestinal bleeding, intractable pain, ureteral obstruction, urinary tract infection, dehydration (from poor oral intake as well as chemotherapy induced diarrhea) and intestinal obstruction. For the patients with unresectable disease, multiple palliative treatment strategies exist which include chemotherapy, chemo-radiation therapy, palliative surgery, fecal diversion, and endoscopic stenting [2]. In addition to improving overall survival, therapy for this patients' population should focus on improving the quality of life. The aim of this study is to determine whether providing chemo-radiation to patients with stage IV rectal cancer will decrease the incidence of cancer related morbidities. 


\section{Methods}

After obtaining IRB approval, a retrospective review of a prospectively maintained database was conducted of all patients who presented with rectal cancer to the John $\mathrm{H}$. Stroger Hospital of Cook County in Chicago from January 2004 to December 2008. Patients were included in the study, if they had clinical, radiologic or pathologic evidence of metastatic rectal cancer (Stage IV). If the primary source of cancer could not be ascertained or determined or there was a history of two or more types of malignancies, patients were excluded from the study. Forty-three patients met all inclusion and exclusion criteria.

At the time of diagnosis, all patients were discussed at a multidisciplinary conference and a treatment plan was formulated for each one. The treatment plans were determined based on the extent of pelvic tumor burden. Patients with T4 tumors, as defined by a fixed tumor on digital rectal examination, radiographic involvement of adjacent organs, vasculature, and sacral nerve roots S1 S2, or the pelvic sidewalls were considered locally advanced. The term "bulky disease" was defined radiographically as $>30 \%$ replacement of the pelvis with tumor. Patients with bulky disease were considered to have locally advanced disease.

Patients with evidence of locally advanced disease received infusional 5-Fluorouracil (5-FU), with or without bevacizumab, and 3-dimensional conformed mega voltage photon therapy totaling 5400-cGy external beam radiation. Patients without evidence of locally advanced or bulky disease received either 5-FU, Leucocorvorin, and Oxaliplatin (FOLFOX) or Leucovorin, and Irinotecan (FOLFIRI) with or without bevacizumab.

Data on demographics, number and location of metastasis, imaging studies, complications, number of blood transfusions, number hospitalization, length of stays and surgical intervention were analyzed using SPSS statistical software. A p value of less than 0.05 was considered statistically significant.

\section{Results}

Between January 2004 to December 2008, 43 patients presented to our institution with stage IV rectal cancer were studied. The mean follow-up was 12 months. In addition to distal metastasis, 18 patients were considered to have locally advanced and/or bulky pelvic disease. This group of patients received combined multi-modality therapy (C-RT) while the remaining 25 who had stage IV rectal cancer without evidence of locally advanced disease or significant pelvic tumor burden received FOLFOX or FOLFIRI with or without bevacizumab (C).

The median age for patients with C-RT group was 50 years (range 27 - 64) and 58.2 years (range 37 - 72 years) for patients in the C group ( $\mathrm{p}=\mathrm{NS})$. The prevalence of males among the patients who received C-RT (77.7\%) was similar ( $\mathrm{p}=\mathrm{NS})$ to the male prevalence in $\mathrm{C}$ group (76\%) (Table 1).

Twenty-five percent of patients developed either clinical or radiographic evidence of at least partial ureteral obstruction. Three patients (12\%) in the C group developed hydronephrosis requiring either internal ureteral stenting or percutaneous nephrostomy tubes compared to 8 patients (44\%) in the C-RT group. The difference in urological intervention was statically significant $(\mathrm{p}<$ 0.05).

Half of all patients in the multi-modality group (9/18) ultimately required proximal diversion due to symptoms of intestinal obstruction. Only $24 \%$ of patients in the chemotherapy group required proximal diversion. Although the need for a stoma occurred more frequently in the C-RT group (50\%), the difference was not statistically significant $(p=0.07)$.

The type of therapy did not influence the rate of transfusion between the two groups. Patients in the C-RT group received approximately 2 units of blood products compared to 1.56 units in the $\mathrm{C}$ group $(\mathrm{p}=\mathrm{NS})$.

The vast majority of patients (72\%) with stage IV rectal cancer, regardless of the treatment regiment, required multiple hospitalizations for intractable pain, bleeding, intestinal obstruction, ureteral obstruction, complications related to chemotherapy and sepsis. Patients who received a multi-modality treatment plan (C-RT) were less likely to be hospitalized on multiple occasions (61\%) compared to patients those who received only chemotherapy $(80 \%)(\mathrm{p}<0.01)$ the overall survival was similar between the two groups $(\mathrm{p}=\mathrm{NS})$ (Table 2).

Table 1. Demographics of patients with stage IV rectal cancer.

\begin{tabular}{cccc}
\hline & C & C-RT & p value \\
\hline $\begin{array}{c}\text { Number of } \\
\text { Patients }\end{array}$ & 25 & 18 & \\
Age Median & $58.2(37-72)$ & $50(27-64)$ & $\mathrm{p}=\mathrm{NS}$ \\
Male Gender & $76 \%$ & $77.8 \%$ & $\mathrm{p}=\mathrm{NS}$ \\
\hline
\end{tabular}

Table 2. Complications, number of hospitalization and survival of patients with stage IV rectal cancer.

\begin{tabular}{cccc}
\hline & C & C-RT & p value \\
\hline Hydronephrosis & $3(12 \%)$ & $8(44 \%)$ & $<0.05$ \\
Ostomy for Obstruction & $6(24 \%)$ & $9(50 \%)$ & $=0.07$ \\
$\begin{array}{c}\text { Number of Transfusions } \\
\text { per Patient }\end{array}$ & 1.56 & 2.0 & $=\mathrm{NS}$ \\
$\begin{array}{c}\text { Multiple Hospitalizations } \\
\text { (2) }\end{array}$ & $20 \%)$ & $11(61 \%)$ & $<0.01$ \\
\hline
\end{tabular}




\section{Discussion}

The management of rectal cancer has dramatically changed of the last 30 years. The use of neo-adjuvant therapy and total mesorectal excision has significantly improved the overall survival for patients diagnosed with rectal cancer [10-13]. The treatment algorithms for patients with potentially curable disease have been well established and effective. Despite maximal treatment, the overall survival for patients with stage IV disease remains poor. Furthermore, many patients spend a significant number of their last days hospitalized for the management of complications related to their disease. The optimal treatment strategies for these patients remain controversial. While many studies have focused on determining which treatment maximizes the overall survival, few have focused on the implication of a recommended treatment on the patients quality life.

It has been well documented [11,14-17] that radiation therapy improves palliation in patients with unresectable rectal cancer with regards to pain and bleeding. But patients may still require frequent hospitalization for the management of other complications. In our series, $72 \%$ of all patients required multiple impatient hospital visits. Fewer patients required multiple hospitalizations when they received a combination of chemotherapy and radiation rather than chemotherapy alone $61 \%$ vs. $80 \%$ (p < 0.01 ). This finding was somewhat surprising because the patients who received multi-modality therapy tended to have a significant tumor burden in the pelvis. This is clearly evidenced by the fact that more patients in the C-RT required proximal fecal diversion ureteral instrumentation, and blood transfusions. However, despite these findings, patients required fewer inpatient hospital visits.

One potential reason for the fewer observed inpatient hospitalizations could be explained by the greater length of stay (LOS) for the C-RT group. The mean LOS was almost twice as long for the patients in the C-RT group (17.3 vs. 8.9 days). However, three patients with extremely advanced disease dramatically influenced the LOS. The mean LOS for these three patients was 78 days. When these three patients are excluded, the LOS for the C-RT group is 5.2 days. While hospice services were suggested for the patients with the most advance disease, these three patients elected to pursue a more aggressive treatment strategy.

Although, formal quality of life surveys were not used in this study, one may infer that less hospital visits could translate into an improved quality of life. Larger prospective randomized studies are needed to investigate and validate this finding.

\section{Conclusion}

Chemo-radiation for patients with locally advanced un- resectable stage IV rectal cancer is not a well established protocol. In our series patients treated with C-RT for bulky stage IV rectal cancer required fewer hospitalizations when compared to stage IV rectal cancer patients treated with chemotherapy alone. In the properly selected patient, fewer hospital visits may improve the quality of life of patients with unresectable stage IV rectal cancer.

\section{REFERENCES}

[1] American Cancer Society, "Colorectal Cancer Facts \& Figures 2011-2013,” American Cancer Society, Atlanta, 2011.

[2] S. M. Ronnekleiv-Kelly and G. D. Kennedy, "Management of Stage IV Rectal Cancer: Palliative Options," World Journal of Gastroenterology, Vol. 17, No. 7, 2011, pp. 835-847. doi:10.3748/wjg.v17.i7.835

[3] A. Jemal, R. Siegel, E. Ward, Y. Hao, J. Xu and M. J. Thun, "Cancer Statistics, 2009," CA: A Cancer Journal for Clinicians, Vol. 59, No. 4, 2009, pp. 225-249. doi:10.3322/caac.20006

[4] R. Stangl, A. Altendorf-Hofmann, R. M. Charnley and J. Scheele, "Factors Influencing the Natural History of Colorectal Liver Metastases,” Lancet, Vol. 343, No. 8910, 1994, pp. 1405-1410. doi:10.1016/S0140-6736(94)92529-1

[5] R. J. Heald and R. D. H. Ryall, "Recurrence and Survival after Total Mesorectal Excision for Rectal Cancer," Lancet, Vol. 327, No. 8496, 1986, pp. 1479-1482. doi:10.1016/S0140-6736(86)91510-2

[6] J. K. MacFarlane, R. D. H. Ryall and R. J. Heald, "Mesorectal Excision for Rectal Cancer,” Lancet, Vol. 341, No. 8843, 1993, pp. 457-460.

doi:10.1016/0140-6736(93)90207-W

[7] Swedish Rectal Cancer Trial, "Improved Survival with Preoperative Radiotherapy in Resectable Rectal Cancer," The New England Journal of Medicine, Vol. 336, No. 14, 1997, pp. 980-987. doi:10.1056/NEJM199704033361402

[8] C. Camma, M. Giunta, F. Fiorica, et al., "Preoperative Radiotherapy for Resectable Rectal Cancer: A Meta-Analysis,” Journal of American Medical Association, Vol. 284, No. 8, 2000, pp. 1008-1015.

doi:10.1001/jama.284.8.1008

[9] A. DeGramont, J. F. Bosset, C. Milan, P. Rougier, O. Bouché, P. L. Etienne, F. Morvan, C. Louvet, T. Guillot, E. François and L. Bedenne, "Randomized Trial Comparing Monthly Low-Dose Leucovorin and Fluorouracil Bolus with Bimonthly High-Dose Leucovorin and Fluorouracil Bolus Plus Continuous Infusion for Advanced Colorectal Cancer: A French Intergroup Study,” Journal of Clinical Oncology, Vol. 15, No. 2, 1997, pp. 808-815.

[10] T. Kramer, R. Share, K. Kiel, et al., "Intraoperative Radiation Therapy of Colorectal Cancer,” In: M. Abe, Ed., Intraoperative Radiation Therapy, Pergamon Press, New York, 1991, pp. 308-310.

[11] I. D. Nagtegaal, C. A. Marijnen, E. K. Kranenbarg, et al., "Circumferential Margin Involvement Is Still an Important Predictor of Local Recurrence in Rectal Carcinoma: 
Not One Millimeter But Two Millimeters Is the Limit," American Journal of Surgical Pathology, Vol. 26, No. 3, 2002, pp. 350-357. doi:10.1097/00000478-200203000-00009

[12] A. Wibe, P. R. Rendedal, E. Svensson, et al., "Prognostic Significance of the Circumferential Resection Margin Following Total Mesorectal Excision for Rectal Cancer,” British Journal of Surgery, Vol. 89, No. 3, 2002, pp. 327334. doi:10.1046/j.0007-1323.2001.02024.x

[13] J. F. Bosset, L. Collette, G. Calais, et al., "Chemotherapy with Preoperative Radiotherapy in Rectal Cancer," The New England Journal of Medicine, Vol. 355, No. 11, 2006, pp. 1114-1123. doi:10.1056/NEJMoa060829

[14] R. K. Wong, V. Tandan, S. De Silva, A. Figueredo, "PreOperative Radiotherapy and Curative Surgery for the Management of Localized Rectal Carcinoma," Cochrane Da- tabase Systematic Reviews, Vol. 2, No. 2, 2007, Article ID: CD 002102.

[15] C. G. Willett and L. L. Gunderson, "Palliative Treatment of Rectal Cancer: Is Radiotherapy Alone a Good Option?” Journal of Gastrointestinal Surgery, Vol. 8, No. 3, 2004, pp. 277-279. doi:10.1016/j.gassur.2003.11.015

[16] S. H. Bae, W. Park, D. H. Choi, H. Nam, W. K. Kang, Y. S. Park, J. O. Park, H. K. Chun, W. Y. Lee, S. H. Yun and H. C. Kim, "Palliative Radiotherapy in Patients with a Symptomatic Pelvic Mass of Metastatic Colorectal Cancer,” Radiation Oncology, Vol. 6, No. 52, 2011, pp. 1-8. doi:10.1186/1748-717X-6-52

[17] A. Suppiah, J. E. Hartley and J. R. Monson, “Advances in Radiotherapy in Operable Rectal Cancer,” Digestive Surgery, Vol. 26, No. 3, 2009, pp. 187-199. doi:10.1159/000219931 